EPJ Web of Conferences 21, 09001 (2012)

DOI: 10.1051/epjconf/20122109001

(c) Owned by the authors, published by EDP Sciences, 2012

\title{
The density of available states of the DDHMS pre-equilibrium model
}

\author{
B. V. Carlson and D. F. Mega ${ }^{\mathrm{a}}$
}

Instituto Tecnológico de Aeronáutica, São José dos Campos SP, Brazil

\begin{abstract}
Griffin's exciton model of pre-equilibrium emission and Blann's hybrid model have proven extremely successful in describing the energy dependence and, to a certain extent the angular dependence, of nucleon and composite particle emission in preequilibrium reactions. However, the conceptual basis of these models was called into question by Bisplinghoff already some time ago. In response to Bisplinghoff, Blann proposed the hybrid Monte Carlo simulation model (HMS), which uses only the densities of available states for creation and decay of single particle-hole pairs. The model was later extended, in collaboration with Chadwick, to the double-differential HMS, which we call the DDHMS. This extension is based on the Chadwick-Obložinský prescription for approximating the energy-angular distribution of available two-particle-one-hole states. Here, we show how this distribution can be calculated exactly.
\end{abstract}

\section{Introduction}

Nuclear reactions were initially classified as either direct or equilibrium processes. In a direct nucleoninduced reaction, the incident nucleon interacts with a target nucleus on a time scale of the order of the time it takes to pass by the target and often excites collective degrees of freedom of the target. A nucleon-induced equilibrium process can be initiated by a direct reaction, but usually involves the absorption of the incident nucleon followed, after a relatively long time, by the emission of other nucleons, composite particles and $\gamma$ rays. This decay process is well described by the statistical theory of nuclear reactions, which assumes that the nucleus reaches internal equilibrium before decaying.

Reactions intermediate between the two also occur, in which particles are emitted before complete equilibrium is reached. Such reactions are called pre-equilíbrium reactions and have been studied extensively. The first model of preequilibrium reactions, the exciton model, was formulated by Griffin in 1966 [1]. This semi-classical model classified nuclear excitations as particles above and holes below the Fermi energy, jointly called excitons. It sought to describe the formation and decay of the nuceus resulting from a nucleon-induced reaction at each stage, until the nucleus reaches equilibrium. The exciton model was extended by Blann [2], by introducing the possibility of emitting charged particles, and by Williams [3], who extended the densities of excitons states and of transitions by including a correction for Pauli blocking and extending the densities to two types of fermions. Another semiclassical model frequently used to describe preequilibrium reactions is the hybrid model, proposed by Blann in 1971 [4]. There, he used the same basic ideas of Griffin to describe the intermediate states together with the description of particle emission and transitions proposed by Harp, Miller and Berne [5] about.

After serious criticisms of the statistical hypotheses used in the exciton and hybrid model were put forth by Bisplinghoff [6], Blann proposed an alternative, which he called the hybrid Monte Carlo simulation (HMS) model [7]. This uses the basic emission and transition rates of the hybrid model

\footnotetext{
${ }^{\text {a }}$ DFM is supported by FAPESP. BVC acknowledges support from FAPESP and the CNPq. e-mail: brett@ita.br
}

This is an Open Access article distributed under the terms of the Creative Commons Attribution-Noncommercial License 3.0, which permits unrestricted use, distribution, and reproduction in any noncommercial medium, provided the original work is properly cited. 
but makes no assumptions concerning the statistical equilibrium of the excited holes and particles. Rather, each of these is treated as an independent excitation, with subsequent emissions and rescatterings followed using a Monte Carlo simulation. We have performed microscopic numerical simulations including transitions rates between all energy-conserving configurations of the nucleus and found the HMS to indeed provide a more faithful description of the stages of a nuclear reaction than do the exciton or hybrid models[8]. An additional advantage of the HMS model compared to earlier models is that it easily permits multiple emissions from the precompound nucleus. Two years later, Blann and Chadwick, generalized the model to the DDHMS (double differential hybrid Monte Carlo simulation)[9] model by including the calculation of angular distributions based on the work of Chadwick and Obložinský[10,11]. We show how the double differental transition densities of the DDHMS can be easily sampled using Monte Carlo methods.

\section{The $1 p \rightarrow 2 p 1 h$ and $1 h \rightarrow 1 p 2 h$ transition densities}

We define the $1 p \rightarrow 2 p 1 h$ transition density by

$$
\begin{array}{r}
\rho_{1 p \rightarrow 2 p 1 h}\left(\mathbf{p}_{\mathbf{1}}\right)=\frac{a V^{2}}{(2 \pi \hbar)^{6}} \int \delta\left(\mathbf{p}_{\mathbf{1}}+\mathbf{p}_{\mathbf{2}}-\mathbf{p}_{\mathbf{3}}-\mathbf{p}_{\mathbf{4}}\right) \delta\left(\frac{p_{1}^{2}}{2 m}+\frac{p_{2}^{2}}{2 m}-\frac{p_{3}^{2}}{2 m}-\frac{p_{4}^{2}}{2 m}\right) \\
\quad \times \theta\left(p_{F 2}-\left|\mathbf{p}_{2}\right|\right) d^{3} p_{2} \theta\left(\left|\mathbf{p}_{\mathbf{3}}\right|-p_{F 1}\right) d^{3} p_{3} \theta\left(\left|\mathbf{p}_{\mathbf{4}}\right|-p_{F 2}\right) d^{3} p_{4},
\end{array}
$$

where $a$ is a symmetry factor of value 1 when the particles are distinct and $1 / 2$ when they are identical and $V$ is the volume. We identify the incident particle 1 with the final particle 3 and the incident particle (final hole) 2 with the final particle 4, by giving each pair the same Fermi momentum. This expression enforces momentum and energy conservation and limits the particle and hole momenta to

$$
\left|\mathbf{p}_{1}\right| \geq p_{F 1}, \quad\left|\mathbf{p}_{2}\right| \leq p_{F 2}, \quad\left|\mathbf{p}_{\mathbf{3}}\right| \geq p_{F 1} \quad \text { e } \quad\left|\mathbf{p}_{\mathbf{4}}\right| \geq p_{F 2} .
$$

To calculate the transition density, we rewrite it in terms of variables that simplify the integrations. We begin by noting that the momenta $\mathbf{p}_{1}$ and $\mathbf{p}_{2}$ can be written as

$$
\mathbf{p}_{10}=U\left(\phi_{12}\right) \frac{p_{1}}{\left|\mathbf{p}_{1}+\mathbf{p}_{2}\right|}\left(\begin{array}{c}
-p_{2} \sin \theta_{12} \\
0 \\
p_{1}+p_{2} \cos \theta_{12}
\end{array}\right) \quad \text { and } \quad \mathbf{p}_{20}=U\left(\phi_{12}\right) \frac{p_{2}}{\left|\mathbf{p}_{1}+\mathbf{p}_{2}\right|}\left(\begin{array}{c}
p_{1} \sin \theta_{12} \\
0 \\
p_{2}+p_{1} \cos \theta_{12}
\end{array}\right) \text {, }
$$

in the frame in which their sum is reduced to a single component along the new $z$ axis,

$$
\mathbf{p}_{10}+\mathbf{p}_{20}=\left(\begin{array}{c}
0 \\
0 \\
\left|\mathbf{p}_{1}+\mathbf{p}_{2}\right|
\end{array}\right)
$$

where $U\left(\phi_{12}\right)$ is an arbitrary rotation about the $z$ axis.

We use these as guides to define $\mathbf{p}_{3}$ and $\mathbf{p}_{4}$ in the frame in which their sum is reduced a single component as

$$
\mathbf{p}_{30}=U\left(\phi_{34}\right) \frac{p_{3}}{\left|\mathbf{p}_{3}+\mathbf{p}_{4}\right|} U_{34}\left(\begin{array}{c}
-p_{4} \sin \theta_{34} \\
0 \\
p_{3}+p_{4} \cos \theta_{34}
\end{array}\right) \quad \text { and } \quad \mathbf{p}_{40}=U\left(\phi_{34}\right) \frac{p_{4}}{\left|\mathbf{p}_{3}+\mathbf{p}_{4}\right|} U_{34}\left(\begin{array}{c}
p_{3} \sin \theta_{34} \\
0 \\
p_{4}+p_{3} \cos \theta_{34}
\end{array}\right) \text {, }
$$

so that

$$
\mathbf{p}_{30}+\mathbf{p}_{40}=\left(\begin{array}{c}
0 \\
0 \\
\left|\mathbf{p}_{3}+\mathbf{p}_{4}\right|
\end{array}\right)
$$




\section{$\mathrm{CNR} * 11$}

where $U\left(\phi_{34}\right)$ is also an arbitrary rotation about the $z$ axis. As the orientation of $\mathbf{p}_{3}+\mathbf{p}_{4}$ with respect to $\mathbf{p}_{1}+\mathbf{p}_{2}$ is arbitrary, we can write their general form as

$$
\mathbf{p}_{3}=U\left(\theta_{x}, \phi_{x}\right) \mathbf{p}_{30} \quad \text { and } \quad \mathbf{p}_{4}=U\left(\theta_{x}, \phi_{x}\right) \mathbf{p}_{40}
$$

where $U\left(\theta_{x}, \phi_{x}\right)$ is a general rotation. It can be shown that

$$
d^{3} p_{3} d^{3} p_{4}=p_{3}^{2} d p_{3} p_{4}^{2} d p_{4} d \cos \theta_{34} d \phi_{34} d \cos \theta_{x} d \phi_{x}
$$

so that this representation can be easily substituted for the original one. For convenience, we also represent the momentum of the particle in the Fermi sea with reference to the incident particle momentum, so that $d^{3} p_{2}=p_{2}^{2} d p_{2} d \cos \theta_{12} d \phi_{12}$.

The integrals can now be evaluated with no great difficulty. We find, when $p_{1} \geq p_{F 2}$,

$$
\begin{aligned}
\rho_{1 p \rightarrow 2 p 1 h}\left(\mathbf{p}_{1}\right)=\frac{a V^{2}}{(2 \pi)^{4} \hbar^{6}} \frac{m}{p_{1}} & \left\{\left(p_{1}^{2}-p_{F 1}^{2}-2 p_{F 2}^{2} / 5\right) p_{F 2}^{3} / 3+2 p_{2 m i n}^{5} / 15\right. \\
& \left.-\theta\left(p_{F 2}-p_{F 1}\right)\left(p_{F 2}-p_{F 1}\right)^{3}\left(p_{F 1}^{2}+3 p_{F 1} p_{F 2}+p_{F 2}^{2}\right) / 15\right\},
\end{aligned}
$$

where $p_{2 \min }^{2}=\max \left(0, p_{F 1}^{2}+p_{F 2}^{2}-p_{1}^{2}\right)$. When $p_{1}<p_{F 2}$, in which case necessarily $p_{F 1}<p_{F 2}$, we find

$$
\rho_{1 p \rightarrow 2 p 1 h}\left(\mathbf{p}_{1}\right)=\frac{a V^{2}}{(2 \pi)^{4} \hbar^{6}} \frac{m}{p_{1}}\left(2 p_{1}^{5}-5 p_{1}^{2} p_{F 1}^{3}+3 p_{F 1}^{5}\right) / 15
$$

The magnitudes and angles of the momenta can be sampled using a Monte Carlo technique based on the partial integrals or an acceptance-rejection algorithm (with rejection due to the Pauli blocking, in the simplest case). We first sample the magnitude $p_{2}$, followed by $p_{3}$, which determines $p_{4}$ through energy conservation. We then sample $\cos \theta_{12}$, which determines $\cos \theta_{34}$ through momentum conservation. The angles $\phi_{12}$ and $\phi_{34}$ can be sampled independently. We assume the initial particle momentum to be along the $z$ axis.

Evaluation of the $1 h \rightarrow 1 p 2 h$ transition density, given by

$$
\begin{array}{r}
\rho_{1 h \rightarrow 1 p 2 h}\left(\mathbf{p}_{\mathbf{1}}\right)=\frac{a V^{2}}{(2 \pi \hbar)^{6}} \int \delta\left(\mathbf{p}_{\mathbf{1}}+\mathbf{p}_{\mathbf{2}}-\mathbf{p}_{\mathbf{3}}-\mathbf{p}_{\mathbf{4}}\right) \delta\left(\frac{p_{1}^{2}}{2 m}+\frac{p_{2}^{2}}{2 m}-\frac{p_{3}^{2}}{2 m}-\frac{p_{4}^{2}}{2 m}\right) \\
\quad \times \theta\left(\left|\mathbf{p}_{2}\right|-p_{F 2}\right) d^{3} p_{2} \theta\left(p_{F 1}-\left|\mathbf{p}_{\mathbf{3}}\right|\right) d^{3} p_{3} \theta\left(p_{F 2}-\left|\mathbf{p}_{\mathbf{4}}\right|\right) d^{3} p_{4},
\end{array}
$$

proceeds in a manner very similar to that of the $1 p \rightarrow 2 p 1 h$ transition density, the principal difference being the limits on the momenta, with $\mathbf{p}_{2}$ now being above the Fermi momentum and $\mathbf{p}_{3}$ and $\mathbf{p}_{4}$ being below it. Using the same procedure as before, we find here, when $p_{1} \leq p_{F 2}$,

$$
\begin{aligned}
\rho_{1 h \rightarrow 1 p 2 h}\left(\mathbf{p}_{1}\right)=\frac{a V^{2} m}{4(2 \pi)^{4} \hbar^{6}}\left\{\left(p_{F 1}^{2}-p_{1}^{2}\right)^{2}\right. & \\
-\theta\left(p_{F 1}-p_{F 2}\right) & {\left[\left(p_{F 1}^{2}-p_{F 2}^{2}\right)^{2}-4 p_{1}^{2}\left(p_{F 1}^{2}-p_{F 2}^{2}\right) / 3\right.} \\
& \left.\left.+8\left(p_{1}^{4}-\left(p_{1}^{2}+p_{F 2}^{2}-p_{F 1}^{2}\right)^{5 / 2} / p_{1}\right) / 15\right]\right\},
\end{aligned}
$$

and when $p_{1}>p_{F 2}$, in which case necessarily $p_{F 1}>p_{F 2}$,

$$
\rho_{1 h \rightarrow 1 p 2 h}\left(\mathbf{p}_{1}\right)=\frac{a V^{2}}{(2 \pi)^{4} \hbar^{6}} \frac{m}{p_{1}}\left\{p_{F 2}^{3}\left(p_{F 1}^{2}-p_{1}^{2}\right) / 3-2\left[p_{F 2}^{5}-\left(p_{1}^{2}+p_{F 2}^{2}-p_{F 1}^{2}\right)^{5 / 2}\right] / 15\right\}
$$




\section{EPJ Web of Conferences}

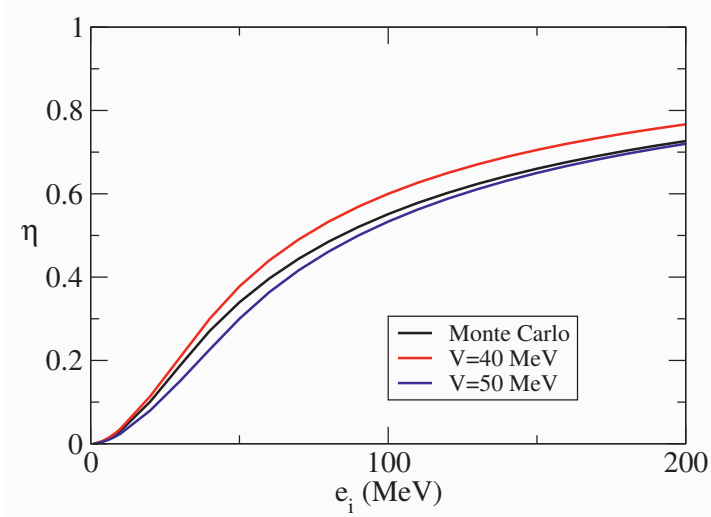

Fig. 1. Reduction in density of $1 p \rightarrow 2 p 1$ transitions due to Pauli blocking as a function of the incident particle energy.

\section{Calculations}

Here we discuss calculations of the $1 p \rightarrow 2 p 1 h$ transition density and particle and hole distributions performed assuming that the two Fermi energies have the same value of $e_{F}=35 \mathrm{MeV}$. We define particle and hole energies, $e_{p}$ and $e_{h}$, with respect to the Fermi energy as

$$
e_{p}=\frac{p_{p}^{2}}{2 m}-e_{F} \quad \text { and } \quad e_{h}=e_{F}-\frac{p_{h}^{2}}{2 m}
$$

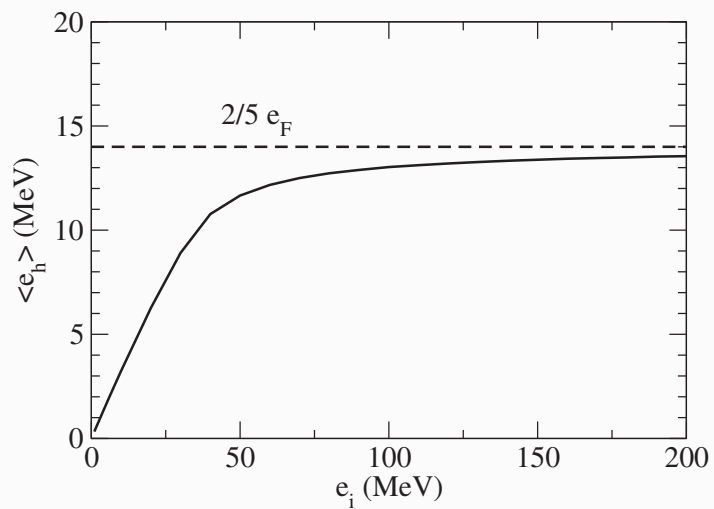

Fig. 2. Average energy of hole produced by $1 p \rightarrow 2 p 1 \mathrm{~h}$ transitions as a function of the incident particle energy.

We begin by presenting the reduction in the density of $1 p \rightarrow 2 p 1 h$ transitions due to the Pauli blocking, which we define as

$$
\eta\left(e_{i}\right)=\rho\left(\mathbf{p}_{i}\right) / \rho_{\text {no-blocking }}\left(\mathbf{p}_{i}\right) .
$$

Kikuchi and Kawai obtained for this quantity [12],

$$
\eta_{K-K}\left(e_{i}\right)=1-\frac{7}{5} r\left(e_{i}\right)+\theta\left(\left(r\left(e_{i}\right)-1 / 2\right) \frac{2}{5} r\left(e_{i}\right)\left(2-1 / r\left(e_{i}\right)\right)^{5 / 2},\right.
$$




\section{CNR*11}

where

$$
r\left(e_{i}\right)=\frac{e_{F}}{e_{F}+e_{i}} .
$$

We find

$$
\eta\left(e_{i}\right)=\frac{\eta_{K-K}\left(e_{i}\right)}{1+\frac{3}{5} r\left(e_{i}\right)},
$$

where we take into account the contribution of the Fermi sea to the unblocked transition density. We compare the two expressions in Fig. 1, in which our reduction factor, with $e_{F}=35 \mathrm{MeV}$, is labeled as Monte Carlo while two evaluations of the Kikuchi-Kawai function are labeled by $V=40 \mathrm{MeV}$ and $V=50 \mathrm{MeV}$, which correspond to the Fermi energies used in their evaluation. It is easy to verify that the Kikuchi-Kawai expression with a Fermi energy of $50 \mathrm{MeV}$ approaches our expression with a Fermi energy of $35 \mathrm{MeV}$ as the incident energy becomes large.

We next look at the average energies of the hole, $\left\langle e_{h}\right\rangle$ and particles $\left\langle e_{p}\right\rangle$ produced by a $1 p \rightarrow$ $2 p 1 h$ transition. When the two Fermi energies are equal, the average energies of the two particles are identical. From energy conservation, we have

$$
\left\langle e_{p}\right\rangle=\frac{e_{i}+\left\langle e_{h}\right\rangle}{2} .
$$

As the incident energy increases, we expect Pauli blocking to become less important and the sampling of the Fermi sea to become more uniform. We thus expect for the average hole energy that

$$
\left\langle e_{h}\right\rangle \rightarrow e_{F}-\langle e\rangle_{\text {Fermi-sea }}=e_{F}-\frac{3}{5} e_{F}=\frac{2}{5} e_{F} \quad \text { as } \quad e_{i} \rightarrow \infty .
$$

This saturation of the average hole energy is seen in Fig. 2, where the average hole energy is already within $10 \%$ of its asymptotic value of $14 \mathrm{MeV}$ when the incident energy is $50 \mathrm{MeV}$.

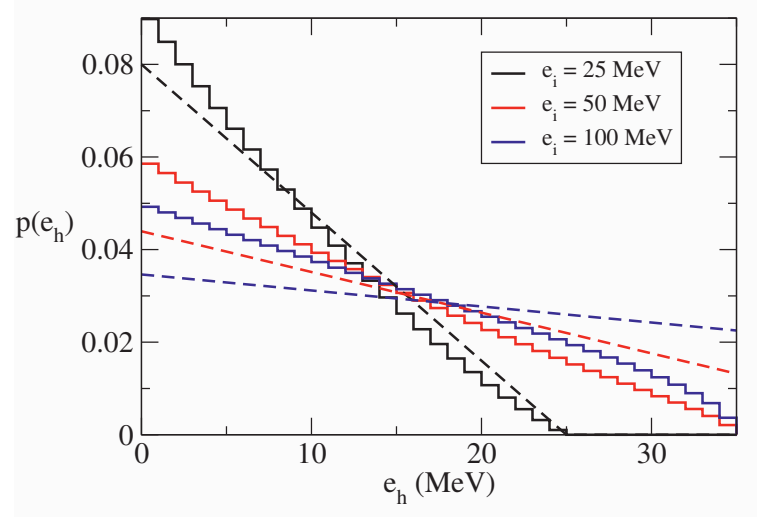

Fig. 3. Energy distribution of holes produced by $1 p \rightarrow 2 p 1 \mathrm{~h}$ transitions at incident particle energies of 25, 50 and $100 \mathrm{MeV}$.

We next discuss the energy and angular distributions of the hole produced in a $1 p \rightarrow 2 p 1 h$ transition. We present the energy and angular distributions of the hole produced in a $1 p \rightarrow 2 p 1 h$ transition in Figs. 3 and 4. In the exciton model, the corresponding hole distribution is given by

$$
p_{e x}\left(e_{h}\right) \propto e_{i}-e_{h},
$$




\section{EPJ Web of Conferences}

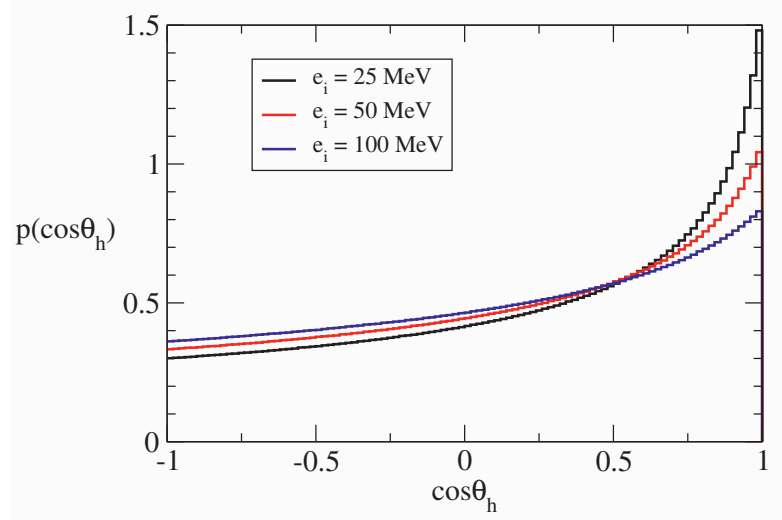

Fig. 4. Angular distribution of holes produced by $1 p \rightarrow 2 p 1 \mathrm{~h}$ transitions at incident particle energies of 25, 50 and $100 \mathrm{MeV}$.

where $e_{i}$ is the energy of the incident particle. The distribution becomes relatively more uniform as the incident particle energy increases, as shown by the dashed line in Fig. 3. In the Fermi gas transition density, given by the solid line in the figure, the hole distribution tends to the density of states,

$$
p\left(e_{h}\right) \propto \sqrt{e_{F}-e_{h}},
$$

as the incident energy increases and the Pauli blocking becomes less important. The increasing uniformity of the sampling of the particles of the Fermi sea with increasing incident energy is also seen in the diminishing forward peaks of the angular distributions shown in Fig. 4.

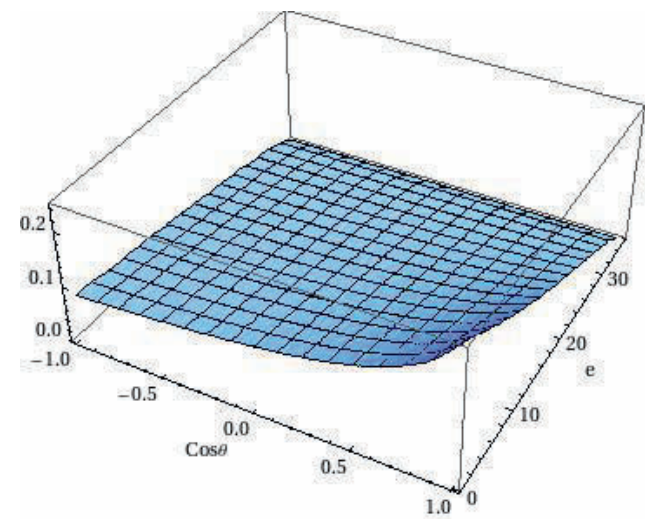

Fig. 5. Energy-angular distribution of holes produced by $1 p \rightarrow 2 p 1 \mathrm{~h}$ transitions at an incident particle energy of $100 \mathrm{MeV}$.

We display the joint energy-angular distribution of the holes produced in a $1 p \rightarrow 2 p 1 h$ transition at an incident particle energy of $100 \mathrm{MeV}$ in Fig. 5. Note that it is isotropic at large hole energy, which corresponds to particle states close to zero in momentum, and becomes more forward peaked as the hole energy decreases and the momentum of the corresponding particle states increase. It thus shows the same general trend as the angular dependence proposed by Chadwick and Obložinský[10, 11 , given for a fixed value of the particle/hole energy $e$ by

$$
p(\theta, e)=\frac{1}{e^{a}-e^{-a}} \exp [a \cos \theta],
$$




\section{CNR*11}

where the parameter $a$ depends on the energy $e$. However, the exact hole angular distribution maintains a more constant value at backwards angles than Chadwick-Obložinský parametrization predicts.

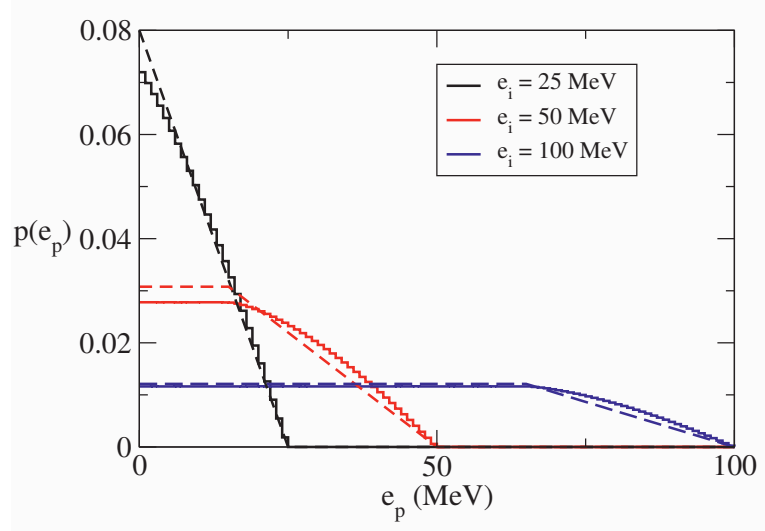

Fig. 6. Energy distribution of particles produced by $1 p \rightarrow 2 p 1 \mathrm{~h}$ transitions at incident particle energies of 25, 50 and $100 \mathrm{MeV}$.

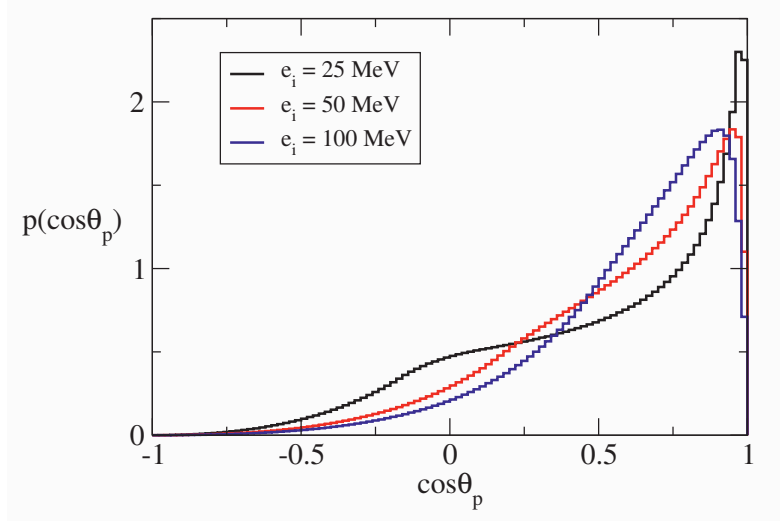

Fig. 7. Angular distribution of particles produced by $1 p \rightarrow 2 p 1 \mathrm{~h}$ transitions at incident particle energies of 25 , 50 and $100 \mathrm{MeV}$.

We present the energy and angular distributions of the particle produced in a $1 p \rightarrow 2 p 1 h$ transition in Figs. 6 and 7. In the exciton model, the particle energy distribution is given by

$$
p_{e x}\left(e_{p}\right) \propto\left\{\begin{array}{cc}
e_{i}-e_{p} & e_{p} \geq e_{i}-e_{F} \\
e_{F} & e_{p} \leq e_{i}-e_{F}
\end{array}\right.
$$

where $e_{i}$ is the energy of the incident particle, as shown by the dashed lines in Fig. 6. The Fermi gas transition density, given by the solid lines in the figure, is very similar, differing in form only at $e_{p}>$ $e_{i}-e_{F}$ and differing slightly in value below this energy due to the difference in form above. The angular distributions, shown in Fig. 7, become more anisotropic as the incident particle energy increases but reach their peaks at nonzero angles, in contrast with the Chadwick-Obložinský parametrization, which 
always peaks at zero degrees. For low values of the incident energy, they also display an angular dependence - a plateau at $\cos \theta \approx 0$ - inconsistent with the exponential dependence on $\cos \theta$ of the Chadwick-Obložinský expression.

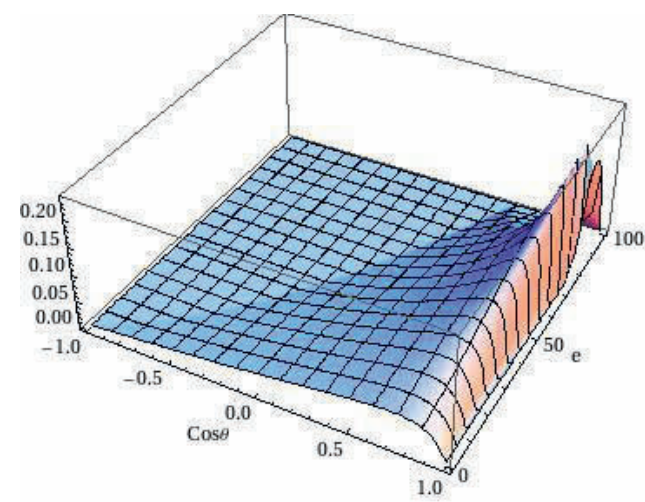

Fig. 8. Energy-angular distribution of particles produced by $1 p \rightarrow 2 p 1 \mathrm{~h}$ transitions at an incident particle energy of $100 \mathrm{MeV}$.

We display the joint energy-angular distribution of the particles produced in a $1 p \rightarrow 2 p 1 h$ transition at an incident particle energy of $100 \mathrm{MeV}$ in Fig. 8. We note immediately that it shows little resemblance to the smooth behavior predicted by the Chadwick-Obložinský angular distribution. It is zero in a large kinematically forbidden region that is restricted to backward angles for low-energy particles but approaches forward angles as the particle energy increases. In fact, one can show that at zero degrees the scattering is limited to the incident energy alone. At lower energies, the distribution peaks at a non-zero forward angle and falls to zero at zero degrees. Although it seems to have been plotted rarely, this distribution was in fact obtained long ago in an analytical form by Kikuchi and Kawai [12].

\section{Conclusion}

We have developed a Monte Carlo algorithm for sampling the two-particle, one-hole energy-angular distribution resulting from a transition induced by a particle in nuclear matter, as well as the oneparticle, two-hole energy-angular distribution resulting from a transition induced by a hole. The algorithm presented here can easily be extended to relativistic kinematics. We find only fair agreement, in the best of cases, between the exact (nonrelativistic) distributions and the usual combination of exciton energy distributions and Chadwick-Obložinský angular distributions used in the DDHMS. We are now implementing the sampling of the exact distribution in DDHMS calculations. However, we have not yet been able to tune the remaining parameters of the model to obtain consistently reasonable results.

\section{References}

1. J. J. Griffin, Phys. Rev. Lett. 17 (1966) 57.

2. M. Blann, Phys. Rev. 21 (1968) 1357.

3. F. C. Williams, Nucl. Phys A166 (1971) 231.

4. M. Blann, Phys. Rev. Lett. 27 (1971) 337.

5. G. D. Harp, J. M. Miller and B. J. Berne, Phys. Rev. 165 (1968) 1166.

6. J. Bisplinghoff, Phys. Rev. C 33 (1986) 1569.

7. M. Blann, Phys. Rev. C 54 (1996) 1341. 


\section{$\mathrm{CNR} * 11$}

8. C. A Soares Pompéia and B. V. Carlson, Phys. Rev. C 74 (2006) 054609.

9. M. Blann and M. B. Chadwick, Phys. Rev. C 57 (1998) 233.

10. M. B. Chadwick and P. Obložinský, Phys, Rev. C 46 (1992) 2028.

11. M. B. Chadwick and P. Obložinský, Phys, Rev. C 50 (1994) 2490.

12. K. Kikuchi and M. Kawai, Nuclear Matter and Nuclear Reactions,(North Holland, Amsterdam, 1968). 